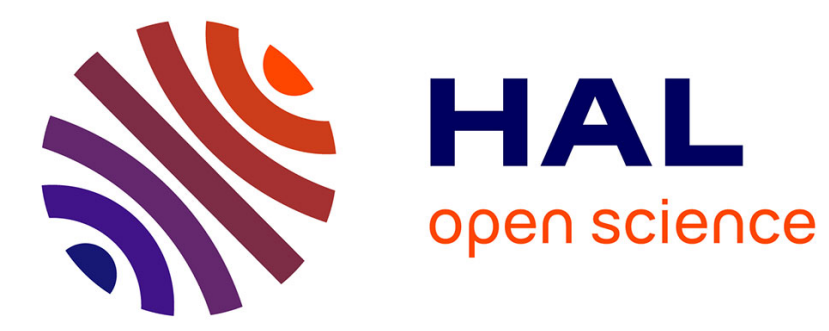

\title{
Partis de gouvernement et transformation des systèmes de clivages
}

Pierre Martin

\section{To cite this version:}

Pierre Martin. Partis de gouvernement et transformation des systèmes de clivages. Pouvoirs - Revue française d'études constitutionnelles et politiques, 2021, 179, pp.59-70. halshs-03404709

\section{HAL Id: halshs-03404709 \\ https://shs.hal.science/halshs-03404709}

Submitted on 26 Oct 2021

HAL is a multi-disciplinary open access archive for the deposit and dissemination of scientific research documents, whether they are published or not. The documents may come from teaching and research institutions in France or abroad, or from public or private research centers.
L'archive ouverte pluridisciplinaire HAL, est destinée au dépôt et à la diffusion de documents scientifiques de niveau recherche, publiés ou non, émanant des établissements d'enseignement et de recherche français ou étrangers, des laboratoires publics ou privés. 


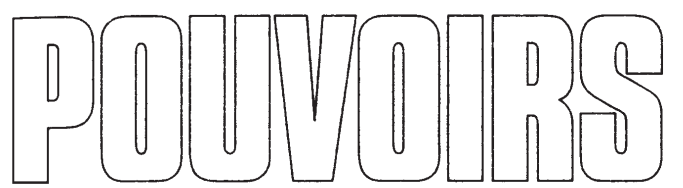

REVUE FRANÇAISE D'ÉTUDES CONSTITUTIONNELLES ET POLITIQUES

LES CLIVAGES

$P \bigcirc L|T| Q \cup E S$

$N^{\circ} \quad 179$ 
Pascal Delwit

Les clivages et la structuration des systèmes partisans

Christophe Le Digol

La permanence du clivage droite/gauche

Olivier Rozenberg

Clivage droite/gauche et $\mathrm{V}^{\mathrm{e}}$ République: une longue tournée d'adieux?

Dominique Reynié

Un clivage fondamental mais sans lendemain

La question européenne en France

Pierre Martin

Partis de gouvernement et transformation des systèmes de clivages

Daniel Boy

Productivistes contre écologistes

Philippe CorcufF

Le progressisme au défi du conservatisme

Manuel Boucher

L'universalisme à l'épreuve des identitaristes décoloniaux 
ISABELle Sommier

Aux antipodes des extrémistes d'hier, les radicaux d'aujourd'hui 103

ÉRIC THIERS

La «pulvérisation démocratique», ou les clivages façon puzzle 115

\section{CHRONIQUES}

Saldi Isra et Hilaire Tegnan

Vers une démocratie présidentielle en Indonésie

\section{REPÈRES ÉTRANGERS}

(1 ${ }^{\text {er }}$ avril - 30 juin 2021)

Pierre Astié, Dominique Breillat et Céline Lageot

CHRONIQUE CONSTITUTIONNELLE FRANÇAISE

(1 ${ }^{\text {er }}$ avril - 30 juin 2021)

Jean Gicquel et Jean-Éric Gicquel

Summaries

www.revue-pouvoirs.fr 


\section{LES CLIVAGES POLITIQUES SONT ÉTUDIÉS PAR}

MANuel Boucher, professeur de sociologie à l'université de Perpignan-Via Domitia. Développant une "sociologie des turbulences", il a notamment publié La Gauche et la Race (L'Harmattan, 2018) et Radicalités identitaires. La démocratie face à la radicalisation islamiste, indigéniste et nationaliste (L'Harmattan, 2020) (manuel.boucher@univ.perp.fr).

Daniel Boy, directeur de recherche émérite au Centre de recherches politiques de l'Iep de Paris (Cevipof). Ses activités de recherche et d'enseignement portaient sur la sociologie électorale, l'analyse des mouvements écologistes en Europe et l'évolution de l'opinion publique vis-à-vis du développement scientifique et technique (daniel.boy@sciencespo.fr).

Philippe CorcufF, maitre de conférences HDR de science politique à l'IEP de Lyon, membre du Centre de recherche sur les liens sociaux (CNRs-Université de Paris-Université Sorbonne Nouvelle). Il a récemment signé La Grande Confusion. Comment l'extrême droite gagne la bataille des idées (Textuel, 2021) (philippe.corcuff@sciencespo-lyon.fr).

Pascal DelWit, professeur de science politique à l'université libre de Bruxelles, membre du Centre d'étude de la vie politique. Spécialiste des partis et des processus électoraux, il est l'auteur des Gauches radicales en Europe, XIX ${ }^{e}-X X I^{e}$ siècles (Éditions de l'université de Bruxelles, 2016) (pascal.delwit@ulb.be).

Christophe Le Digol, maittre de conférences en science politique à l'université Paris Nanterre. Il a en particulier publié Gauche-droite: la fin d'un clivage? Sociologie d'une révolution symbolique (Le Bord de l'eau, 2018) (cledigol@parisnanterre.fr).

Pierre Martin, politologue, chercheur associé au laboratoire Pacte de l'ieP de Grenoble. Il est l'auteur de Crise mondiale et systèmes partisans (Presses de Sciences Po, 2018).

DominiQue Reynié, agrégé de science politique, professeur des universités à l'IEP de Paris, directeur général de la Fondation pour l'innovation politique.

Olivier Rozenberg, professeur associé à l'iep de Paris, membre du Centre d'études européennes et de politique comparée. Il a publié Les Députés français et l'Europe. Tristes hémicycles? (Presses de Sciences Po, 2018).

IsABELLE SOMMIER, professeure de sociologie à l'université Paris 1 Panthéon-Sorbonne, chercheure au Centre européen de sociologie et de science politique (Paris 1-CNRSEHESS). Elle a récemment dirigé, avec la collaboration de Xavier Crettiez et François Audigier, Violences politiques en France, de 1986 à nos jours (Presses de Sciences Po, 2021).

ÉRIC ThIERs, chercheur associé au Cevipof. Il a notamment dirigé, avec Olivier Rozenberg, un Traité d'études parlementaires (Larcier, 2018). 


\section{PARTIS DE GOUVERNEMENT ET TRANSFORMATION DES SYSTÈMES DE CLIVAGES}

n observe à partir des années 1970 un déclin électoral des grands partis de gouvernement en Europe occidentale et l'apparition de nouvelles questions politiques, de nouveaux enjeux, pouvant donner lieu à la formation de nouveaux clivages partisans et à l'émergence de nouvelles forces politiques. Il est ainsi légitime de s'interroger sur les liens entre ces différents phénomènes. Plus précisément, un lien de causalité peut-il être envisagé entre le déclin des partis de gouvernement et l'apparition de nouvelles questions politiques depuis un demi-siècle?

LE DÉCLIN DES GRANDS PARTIS

EN EUROPE OCCIDENTALE

L'Europe occidentale connaît un net déclin électoral des grands partis de gouvernement, sociaux-démocrates (et travaillistes) à gauche, chrétiensdémocrates et conservateurs à droite. Ce déclin s'observe à la fois au niveau de l'ensemble de ces familles politiques, les sociaux-démocrates reculant globalement de $30,5 \%$ à $18,5 \%$ et l'ensemble de la droite modérée de $54,5 \%$ à 40,2\% entre 1970 et 2020, et au niveau individuel de chaque grand parti de gouvernement, avec une symétrie remarquable des reculs entre partis de droite et de gauche d'environ quinze points ${ }^{1}$. Comme ces grands partis disposaient d'une influence électorale structurée par des affrontements politiques sur des enjeux et des clivages

1. Pierre Martin, Crise mondiale et systèmes partisans, Paris, Presses de Sciences Po, 2018, p. 33 (chiffres actualisés jusqu'à fin 2020 par l'auteur).

P O U V O I R - 179.2021 
partisans traditionnels, pour l'essentiel fixés dans les années 1920, il est naturel de penser qu'ils ont en premier lieu été électoralement victimes de l'apparition de nouvelles questions politiques.

Cependant, les choses ne sont pas aussi simples. Les avantages considérables qu'ont les grands partis de gouvernement par rapport à leurs concurrents peuvent leur permettre de faire face à des transformations des demandes des électeurs et de s'adapter à un nouveau paysage politique, à travers de véritables reconversions partisanes (comme les entreprises qui se reconvertissent). Ils ont beaucoup plus de moyens financiers, ce qui leur donne accès aux meilleurs spécialistes de l'opinion (sondeurs), de la communication ainsi qu'à ceux de l'analyse des évolutions de la société. Grâce à leur monopole sur les principaux postes de pouvoir politique, et souvent administratif, ils attirent dans leur rang les meilleurs candidats aux élections. Leurs positions de pouvoir leur permettent également d'être les maîtres du temps et des règles en contrôlant l'agenda politique (parfois même électoral) et les lois électorales. Ils ont enfin une capacité d'influence médiatique supérieure à leurs concurrents, ce qui peut entre autres faciliter la récupération à leur profit d'une nouvelle question politique initialement popularisée par un petit parti (l'environnement ou l'immigration, par exemple).

En un demi-siècle, cette situation dominante leur a permis de développer, souvent avec succès, différents types de stratégies face à l'arrivée de nouveaux concurrents. La première stratégie consiste à diaboliser le nouveau venu (droite radicale) ou à le ridiculiser (écologiste) tout en reprenant ses revendications sous une forme plus modérée. Cette stratégie a été plus particulièrement mise en œuvre dans les pays à système électoral majoritaire. La deuxième stratégie consiste à passer une alliance gouvernementale avec le nouveau parti contestataire pour le banaliser, exposer son inexpérience tout en se positionnant sur le nouvel enjeu. Cette stratégie a souvent été mise en œuvre dans les pays à système électoral très proportionnel. On a ainsi vu la droite modérée faire alliance avec la droite radicale en Autriche (1999, 2017), en Norvège (2013), en Finlande (2015), en Belgique (2014), au Danemark (2015), et les écologistes être introduits au sein du gouvernement par les socialistes, la droite modérée ou les deux en France (1997, 2012), en Allemagne (1998), en Belgique (1999, 2020), en Irlande (2007, 2020), en Islande $(2009,2017)$, au Luxembourg (2013), en Suède (2014), en Finlande $(2007,2019)$ et en Autriche (2019). Ces expériences ont souvent usé les nouveaux venus. Au final, ces partis écologistes ou de droite radicale ne sont jamais devenus électoralement plus forts que 
les anciens partis de gouvernement (sauf l'Union démocratique du centre en Suisse).

DU DÉCLIN DES PARTIS DE GOUVERNEMENT

À L’AFFAIBLISSEMENT DES SYSTĖMES POLITIQUES

Le déclin des grands partis de gouvernement n'est pas le seul phénomène électoral fort et durable que l'on observe partout en Europe occidentale depuis les années 1970: on constate également, de manière concomitante, une progression de la volatilité électorale et un recul de la participation électorale ${ }^{2}$. Or, si l'apparition de nouveaux enjeux peut expliquer le deuxième phénomène, ce n'est absolument pas le cas pour le troisième. Il y a d'autres facteurs fondamentaux qui expliquent ces trois phénomènes électoraux simultanés en Europe occidentale (recul des partis de gouvernement, augmentation de la volatilité électorale et baisse de la participation): le déclin de la croissance économique, les transformations sociétales dues au développement d'une nouvelle forme d'individualisme, la progression de la bureaucratisation et la constitution d'une classe politique.

La compréhension de ces phénomènes est d'autant plus importante que les trois évolutions électorales observées aboutissent à une crise des systèmes politiques occidentaux à travers l'affaiblissement de leur capacité à prendre des décisions légitimes. En effet, le recul de la participation affaiblit la légitimité des élus, celui des grands partis rend plus difficile la formation des gouvernements, nécessitant des coalitions avec un nombre accru d'acteurs politiques, et, enfin, l'accroissement de la volatilité électorale rend l'avenir plus incertain pour les acteurs politiques.

LES TRANSFORMATIONS ÉCONOMIQUES ET SOCIÉTALES ET LEURS CONSÉQUENCES POLITIQUES

Le déclin continu de la croissance économique en Europe occidentale à partir de 1973, passant en moyenne de 4,8\% (taux de croissance annuel du PIB en volume) entre 1950 et 1973 à 2,4\% de 1974 à 2007, puis quasi nulle de 2008 à 2021, est un élément majeur d'explication du recul des grands partis de gouvernement. Il affecte en effet directement la capacité budgétaire des gouvernements, qui ont de plus en plus de difficultés à satisfaire les demandes des citoyens.

2. Id. 
Or ces demandes sont allées croissant au fil du temps. Tout d'abord, la forte progression de l'intervention de l'État, en particulier depuis 1945, a été rendue nécessaire par le développement de la marchandisation liée au capitalisme. Cette intervention a pris diverses formes dont celle du développement de l'État social. De plus, avec la baisse de la croissance et la crise du keynésianisme à partir des années 1970, les demandes d'aide sociale (notamment concernant le chômage) ont été de plus en plus importantes, à l'instar d'autres dépenses, en particulier en termes de santé. Ainsi, l'action des gouvernants n'a pu que susciter des déceptions successives parmi les citoyens, ce qui s'est traduit chez eux par une tendance à sanctionner électoralement les partis au pouvoir.

Les grandes transformations sociologiques et culturelles liées à la prospérité des années 1950-1960, à la forte progression du niveau d'instruction, au développement de la société de consommation et à l'individualisation des comportements ont été une autre source de difficultés pour les gouvernements occidentaux. Ces transformations sociales et culturelles majeures ${ }^{3}$ ont correspondu à une nouvelle vague de sécularisation (effondrement de la pratique religieuse dans les pays européens), ainsi qu'à une exigence visant à plus de transparence, de participation et de liberté chez les jeunes et dans les milieux favorisés, ce qui a contribué au développement de comportements contestataires et de médias critiques. Ces évolutions de long terme ont rendu de plus en plus difficiles la gestion des attentes des populations, situation mise en évidence dès 1975 dans un rapport de la Commission trilatérale ${ }^{4}$. Elles ont également correspondu au développement d'un comportement consumériste y compris dans la sphère publique, à l'affirmation d'un individualisme compétitif (sinon narcissique) favorisant le déclin des solidarités, notamment fiscales, et au renforcement croissant de revendications identitaires intransigeantes, qui ont complexifié les stratégies d'arbitrages des grandes formations politiques et stimulé l'apparition de partis politiques centrés sur un enjeu particulier (écologie, féminisme, immigration, retraites, défense des animaux ou de la chasse) $)^{5}$.

3. Cf. Russell J. Dalton, Democratic Challenges, Democratic Choices: The Erosion of Political Support in Advanced Industrial Democracies, Oxford, Oxford University Press, 2004; Ronald Inglehart, The Silent Revolution: Changing Values and Political Styles Among Western Publics, Princeton (N. J.), Princeton University Press, 1977.

4. Michel Crozier, Samuel P. Huntington et Joji Watanuki, The Crisis of Democracy: Report on the Governability of Democracies to the Trilateral Commission, New York (N. Y.), New York University Press, 1975.

5. Cf. Wolfgang Streeck, «Citizens as Customers », New Left Review, n 76, 2012, p. 27-47. 
Deux autres éléments importants ont enfin, sans doute à un moindre degré, contribué à l'affaiblissement des partis de gouvernement: la prolifération bureaucratique et le développement d'une classe politique. La bureaucratisation du monde ainsi que sa judiciarisation sont des éléments consubstantiels au capitalisme. Elles se sont accrues avec le passage au néolibéralisme à partir des années $1980^{6}$, provoquant des demandes récurrentes de simplification et une lassitude croissante des citoyens. Le développement d'une classe politique au sein des pays occidentaux correspond au déclin de la "politique de classe» dans laquelle les élus étaient fortement liés à la représentation parlementaire de classes sociales (classe ouvrière, bourgeoisie), même s'ils n'en étaient pas toujours originaires, ainsi qu'à l'homogénéisation de la formation intellectuelle des élus (et des journalistes) par l'augmentation générale du niveau d'instruction et par l'accroissement considérable à partir des années 1970 du personnel des collaborateurs d'élus. Un nombre croissant de personnes se mettent à vivre de la politique, et pas seulement pour la politique. Elles sont majoritairement affiliées à des partis de gouvernement dont la concurrence électorale s'accompagne de plus en plus souvent d'une «solidarité systémique» qui les apparente à une sorte de cartel partisan. Ainsi ce groupe de personnes vivant de la politique, lié aux partis de gouvernement, a-t-il naturellement tendance à utiliser le pouvoir politique à son profit (en tant que classe politique) pour se protéger face à l'opinion et à la justice, accroissant en retour la désaffection de l'opinion vis-à-vis du pouvoir politique et des partis de gouvernement ${ }^{7}$.

\section{LA TRANSFORMATION DES SYSTÈmES DE CLIVAGES}

Il est tout d'abord nécessaire de préciser le sens du mot «clivage». Je vais utiliser ici le terme au sens restreint de la théorie des clivages de Lipset et Rokkan précisé par Bartolini ${ }^{8}$.

6. Cf. Béatrice Hibou, La Bureaucratisation du monde à l'ère néolibérale, Paris, La Découverte, 2012.

7. Cf. Jens Borchert et Jürgen Zeiss (dir.), The Political Class in Advanced Democracies: A Comparative Handbook, Oxford, Oxford University Press, 2003; Peter Oborne, Le Triomphe de la classe politique anglaise (2007), Paris, Michalon, 2014.

8. Cf. Seymour Martin Lipset et Stein Rokkan, Party Systems and Voter Alignments: CrossNational Perspectives, New York (N. Y.), Free Press, 1967; Stefano Bartolini, «La formation des clivages », Revue internationale de politique comparée, vol. 12, n 1, 2005, p. 9-34. Voir aussi, supra, Pascal Delwit, «Les clivages et la structuration des systèmes partisans », p. 5-16. 


\section{La théorie des clivages et l'opposition droite/gauche}

Cette théorie explique la formation des systèmes partisans d'Europe occidentale du XIX ${ }^{\mathrm{e}}$ siècle au début du XXe, lors de la construction des États-nations par leurs élites nationales, sous l'action de deux révolutions (la révolution nationale et la révolution industrielle) donnant chacune lieu au développement de deux clivages (centre/périphérie et Église/État, puis rural/urbain et ouvriers/patronat). Dans ce cadre, la singularité de chaque système partisan national correspond aux différents types d'alliances locales que les élites nationales ont faites lors de ces deux révolutions et au poids spécifique de chacun de ces clivages. Ces systèmes partisans ont été globalement stables depuis les années 1920. Lipset et Rokkan précisent également que ces clivages se sont formés «lors de mouvements de protestation contre l'élite nationale en place et ses standards culturels». Deux remarques s'imposent immédiatement: l'opposition droite/gauche n'est pas un clivage, et le rôle des élites est incontournable dans la formation des clivages.

L'opposition droite/gauche chère aux politistes français n'est pas un clivage, car c'est une opposition dualiste qui a pour fonction de structurer, de rendre lisible, l'ensemble du paysage politique. Un clivage est plus limité dans son extension et plus durable dans son contenu. C'est pourquoi on peut considérer, si l'on veut articuler les deux concepts, que l'opposition droite/gauche évolue au fil du temps selon la pertinence des clivages qui la structurent. Des clivages par rapport auxquels il est à chaque fois possible de définir une position de gauche opposée à une position de droite. Ainsi, sous la IVe République, on peut considérer, en s'inspirant du travail de Maurice Duverger ${ }^{9}$, que deux clivages dominaient la vie politique: Église/État et ouvriers/patronat. Sur chacun de ces clivages, il y avait une position de gauche - État (laicité) et ouvriers (État social) - et de droite - cléricalisme et patronat (libéralisme économique). Les partis de gauche, socialistes et communistes, avaient des positions de gauche sur les deux clivages, les partis de droite, indépendants et gaullistes, des positions de droite, et les partis du centre se définissaient par des positions mixtes, mais opposées, les démocrateschrétiens du MRP (Mouvement républicain populaire) étant cléricaux et sociaux, les radicaux étant laïques et libéraux.

\footnotetext{
9. Les Partis politiques (1951), Paris, Armand Colin, 1973, p. 262.
} 


\section{Nouvelles élites, nouveaux enjeux et nouveaux clivages}

La question des élites est incontournable pour l'étude de la transformation des systèmes de clivages étant donné que leur rôle est fondamental dans la formation des clivages sous deux aspects. Le premier, nous l'avons vu, c'est le rôle des élites nationales en place contre lesquelles s'effectue la mobilisation aboutissant à la formation d'un clivage. Mais, pour que cette mobilisation réussisse, elle doit elle-même être menée par d'autres élites, des contre-élites, des entrepreneurs politiques capables de mettre en place une structure organisationnelle à même de faire accéder un conflit particulier à la scène politique. C'est pourquoi la question de l'actualisation de la théorie des clivages nécessite l'analyse de la transformation des élites dans les pays occidentaux.

Les mutations économiques, politiques, sociales et culturelles dont les pays d'Europe occidentale ont été le théâtre depuis la fin de la Seconde Guerre mondiale ont conduit à de profondes transformations de leurs élites à partir des années 1980. Ces transformations des élites dirigeantes occidentales ont été observées et analysées comme l'apparition d'une nouvelle classe capitaliste transnationale, d'une nouvelle aristocratie, d'un bloc oligarchique néolibéral et de nouvelles élites mondialisatrices ${ }^{10}$, qui ont pour objectif, non plus la construction des États-nations, mais la gestion de la mondialisation. Ces nouvelles élites, qui sont pour beaucoup une transformation des anciennes élites nationales occidentales, ont quatre composantes, d'ordre économique (managers et actionnaires des entreprises multinationales, dont les banques), étatique (responsables politiques et administratifs), professionnel (en particulier juristes, avocats d'affaires, conseillers fiscaux, élites universitaires et médicales) et consumériste (direction des entreprises de communication et de commerce). D'origine et de composition principalement occidentales (en particulier anglo-saxonnes), ces nouvelles élites dirigeantes se caractérisent par le libéralisme politique et culturel, le nomadisme, la valorisation de la concurrence, un productivisme qui s'accompagne cependant d'une adhésion au concept de développement durable et d'une volonté de réduire les inégalités se traduisant essentiellement par la lutte contre les discriminations. Des valeurs sensiblement différentes de celles des élites occidentales dominantes précédentes, qui, si elles adhéraient au

10. Cf. respectivement Leslie Sklair, The Transnational Capitalist Class, Oxford, Blackwell, 2001 ; Lionel Jospin, Le Monde comme je le vois, Paris, Gallimard, 2005; Pierre Dardot et Christian Laval, Ce cauchemar qui n'en finit pas. Comment le néolibéralisme défait la démocratie, Paris, La Découverte, 2016; Pierre Martin, Crise mondiale et systèmes partisans, op. cit. 
libéralisme politique, se réclamaient du conservatisme sociétal et moral traditionnel, valorisaient l'identité nationale et manifestaient un productivisme et un libéralisme économique infléchis après-guerre par la mise en place d'un État social, sous l'influence d'un mouvement ouvrier priorisant l'augmentation des salaires et des droits des travailleurs plutôt que des minorités.

Dès les années 1970, de nouveaux enjeux liés aux transformations culturelles (féminisme, écologie, droits des homosexuels) sont apparus, en opposition aux standards culturels des élites traditionnelles. Ils ont dans un premier temps favorisé le développement de partis de la «nouvelle gauche », mais ont été par la suite intégrés dans les valeurs des nouvelles élites dirigeantes et les programmes des partis sociaux-démocrates et libéraux. Durant cette décennie et la suivante, la contestation des politiques fiscales et du développement de l'État social a trouvé un écho croissant chez les milieux aisés et les classes moyennes supérieures, de plus en plus individualistes, profitant aux partis libéraux et conservateurs dans l'opposition avant d'être intégrée par les grands partis de gouvernement lors de leur ralliement général au néolibéralisme.

En revanche, à partir des années 1980-1990, l'opposition à l'immigration extra-européenne, souvent associée à l'insécurité puis au terrorisme islamique, a conduit à l'apparition d'un clivage identité/cosmopolitisme ${ }^{11}$ et de partis de droite radicale anti-immigration, valorisant l'identité nationale et hostiles à l'intégration européenne. Ces partis ont pu consolider peu à peu leur influence électorale en progressant dans les milieux populaires, en particulier ouvriers, victimes économiques de la mondialisation (libre-échange, concurrence de l'immigration) et attachés aux valeurs culturelles traditionnelles.

On doit aussi envisager l'émergence éventuelle d'un second clivage, de nature plus économique, en réaction au creusement des inégalités salariales et patrimoniales, opposant de nouvelles couches sociales

11. Appelé aussi universalistes/anti-universalistes par Gérard Grunberg et Étienne Schweisguth, «Recompositions idéologiques», in Daniel Boy et Nonna Mayer (dir.), L'électeur a ses raisons, Paris, Presses de Sciences Po, 1997, p. 139-178; ethnocentrisme/cosmopolitisme par André-Paul Frognier, «Partis et clivages en Belgique. L’héritage de S. M. Lipset et S. Rokkan », in Pascal Delwit et Jean-Michel De Waele (dir.), Les Partis politiques en Belgique, Bruxelles, Éditions de l'université de Bruxelles, 1997, p. 249-257; ouverts/fermés par Jean Chiche et al., «L'espace politique des électeurs français à la fin des années 1990. Nouveaux et anciens clivages, hétérogénéité des électorats", Revue française de science politique, vol. 50, n 3, 2000, p. 463-487; intégration/démarcation par Hanspeter Kriesi et al., West European Politics in the Age of Globalization, Cambridge, Cambridge University Press, 2008. 
diplômées insatisfaites de leur situation économique précaire, particulièrement depuis la crise de 2008, aux élites mondialisatrices en place. Ce nouveau clivage, que j'ai appelé altermondialistes/néolibéraux ${ }^{12}$, pourrait expliquer la percée, à partir de 2010, de forces politiques contestataires relevant de la gauche radicale (Syriza en Grèce, Podemos en Espagne, La France insoumise, Sinn Féin en Irlande, socialistes de Bernie Sanders aux États-Unis, corbynistes au Royaume-Uni) ou plus difficilement classables (Partis pirates en Islande et au Luxembourg, Mouvement 5 Étoiles en Italie).

\section{Dynamiques institutionnelles, polarisation et dépolitisation}

Il faut dès maintenant noter que les États-Unis constituent une exception spectaculaire aux trois phénomènes précédemment décrits: les deux grands partis, démocrate et républicain, dominent encore la vie politique de manière quasi exclusive, la participation électorale n'a nullement décliné depuis les années 1970 et la volatilité électorale est très faible et même en recul, les deux camps étant à la fois compacts et stables. Plutôt qu'une aberration, les États-Unis sont un cas limite d'un type de système politique dont on retrouve l'écho dans certains pays européens, notamment ceux pratiquant le scrutin majoritaire (Royaume-Uni, France), où les grandes transformations économiques et sociétales mentionnées plus haut ont des conséquences électorales différentes de celles constatées dans la plupart des autres pays européens.

En effet, deux modèles (des idéaux-types) de système politique au sein des démocraties représentatives occidentales se distinguent de plus en plus sous l'action de différents facteurs analysés auparavant (principalement les transformations économiques, les mutations culturelles, l'apparition de nouveaux enjeux et clivages): dualisme ultra-polarisé et émiettement dépolitisé. Dans le premier modèle, que les États-Unis illustrent presque à la perfection, au sein d'un système institutionnel présentant de puissantes contraintes partisanes (notamment mode de scrutin majoritaire et présidentialisation du régime) qui verrouillent la représentation au profit de deux grands partis, les dynamiques électorales observées dans la majorité des pays d'Europe occidentale depuis 1970 (déclin des grands partis de gouvernement, de la participation, augmentation de la volatilité électorale) sont inversées. Et ce, en raison d'une intense politisation sur les enjeux culturels à fortes valeurs morales (avortement, racisme, féminisme, droits des homosexuels) provoquant

12. Crise mondiale et systèmes partisans, op. cit., p. 227-232. 
de véritables guerres culturelles, alliée à une importante personnalisation des affrontements où chaque camp se mobilise sur la détestation de l'adversaire, situation souvent qualifiée de «politique de haine». À l'opposé, dans le second modèle, représenté par de nombreux systèmes politiques d'Europe occidentale où le mode de scrutin est très proportionnel et le régime exclusivement parlementaire, les trois dynamiques électorales se concrétisent pleinement. Ces systèmes politiques tendent à émietter la représentation parlementaire, la survie de petites formations centrées sur un enjeu étant favorisée par l'individualisme «nombriliste» croissant des électeurs et par la segmentation thématique des médias et des campagnes de communication. On observe également dans les pays en question une évolution de fond vers la dépolitisation et la dépolarisation, les grands partis et leurs leaders se présentant de plus en plus comme des responsables compétents, pragmatiques et désidéologisés ${ }^{13}$. Il faut noter que certains pays ouest-européens, en particulier le Royaume-Uni, la France et l'Italie, qui pour sa part a recours à un mode de scrutin à composante majoritaire, sont des cas mixtes; ils présentent des effets qui caractérisent les deux modèles: polarisation et personnalisation, typiques du premier; mais aussi recul des grands partis et de la participation, accroissement de la volatilité électorale, significatifs du second.

Cependant, aucun de ces deux modèles, dualisme ultra-polarisé et émiettement dépolitisé, n'échappe à la tendance au déclin de la capacité du pouvoir politique à prendre des décisions légitimes. Nous l'avons déjà évoqué pour le second modèle, de par le double recul des grands partis et de la participation électorale combiné à l'augmentation de la volatilité électorale. Mais, dans le premier modèle, l'extrême polarisation risque de provoquer, comme aux États-Unis lors de l'élection présidentielle de 2020, la non-reconnaissance de la légitimité du vainqueur par le camp défait. Elle peut aussi aboutir à une paralysie croissante des institutions parlementaires (tel le Congrès des États-Unis), ainsi qu'à des manifestations violentes contre le pouvoir (à l'exemple des «gilets jaunes» en France en 2018-2019).

13. Comme l'illustre la boutade attribuée au Premier ministre des Pays-Bas, Mark Rutte: «Si vous cherchez une vision, allez chez l'opticien» (Le Journal du dimanche, 14 mars 2021). 
Si le déclin des partis de gouvernement en Europe occidentale depuis les années 1970 est pour une part lié à l'apparition de nouveaux enjeux et de nouveaux clivages, il résulte principalement $d u$ recul de la croissance économique, de l'augmentation des attentes de la population envers l'État et $d u$ développement de comportements contestataires. Ces transformations économiques et sociales ont fortement affaibli la capacité des gouvernants à prendre des décisions légitimes aux yeux des citoyens. 


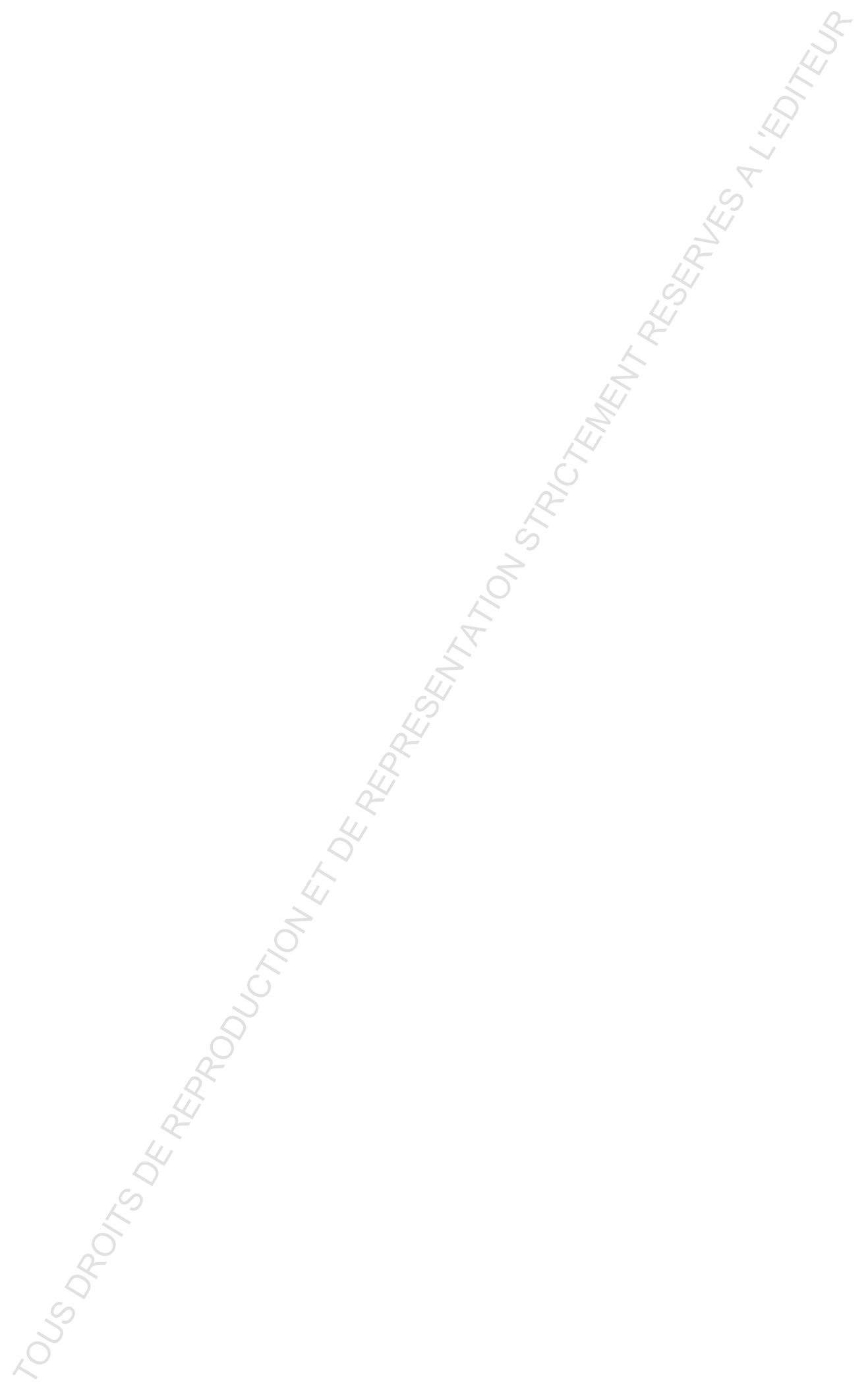

